\title{
Variations des capacités reproductrices de l'escargot " Petit-gris ", Helix aspersa Müller (Mollusque Gastéropode Pulmoné Stylommatophore), selon son origine géographique. \\ II. Incubation des œufs et éclosion des jeunes.
}

\author{
D. GUÉMÉNÉ et J. DAGUZAN \\ U.E.R. des Sciences de la Vie et de l'Environnement, Université de Rennes I \\ Laboratoire de Zoologie générale et d'Ecophysiologie \\ Avenue du Général-Leclerc, F 35042 Rennes Cedex
}

\begin{abstract}
Résumé
Cette étude montre que les durées d'incubation des oufs et d'éclosion des jeunes chez l'escargot Petit-gris, Helix aspersa (Müller), ne dépend, ni de la provenance géographique des reproducteurs, ni du type d'éclairement auquel ces derniers ont été soumis. Il semble que les conditions optimales pour réaliser cette phase de l'élevage soient une température de $20^{\circ} \mathrm{C}$ et une humidité relative de $100 \mathrm{p} .100$. La présence de substrat pour l'incubation (terreau horticole) ne paraît pas être obligatoire et en son absence les durées d'incubation et d'éclosion sont nettement plus courtes.
\end{abstract}

\section{Introduction}

Les durées d'incubation des œufs et d'éclosion des jeunes chez les Gastéropodes Pulmonés sont très mal connues. Carrik (1942) a montré, chez Agriolimax sp., qu'elles varient énormément avec la température, et que loptimum, pour cette espèce est de $20^{\circ} \mathrm{C}$. Chez Helix sp., Basinger (1931), Herzberg \& Herzberg (1962), Pollard (1975) constatent que les fortes températures diminuent le temps d'incubation tandis que les basses températures l'augmentent. De même, ces auteurs notent que la sécheresse retarde l'éclosion des æufs. Ces renseignements, bien que précieux, ne sont pas chiffrés, et sont, de ce fait, inutilisables pour l'heliciculture.

Par contre, Charrier (1980) montre que pour des individus Helix aspersa élevés en bâtiment à $20^{\circ} \mathrm{C}$, la durée d'incubation oscille entre 19 et 22 jours. Récemment, Daguzan (1981) enregistre, au niveau d'un élevage expérimental de 1000 escargots Petits-gris adultes, réalisé en bâtiment et en conditions thermo-hygrométriques contrôlées $\left(20^{\circ} \mathrm{C}, 80\right.$ p. $100 \mathrm{Hr}$; photo-période : 12 heures de jour / 12 heures de nuit), que les durées d'incubation des œufs et d'éclosion des nouveau-nés sont respec- 
tivement de $22,4 \pm 4,6$ jours et de $12,5 \pm 4,8$ jours (1). Enfin, chez l'escargot de Bourgogne (Helix pomatia L.) élevé à $20^{\circ} \mathrm{C}$, la durée moyenne d'incubation difffère selon qu'il s'agit de pontes obtenues au laboratoire (16,5 jours) ou provenant de la nature (18 à 24 jours) (VINCENT et al., 1982).

Comme l'ont affirmé Bagnard \& Bondols (1976), il serait intéressant de savoir s'il est possible de raccourcir le temps d'incubation en utilisant une température et une humidité appropriées. Pour cette raison, à la suite de l'étude que nous avions réalisée récemment sur l'accouplement et la ponte (GuEMENE \& DaguZaN, 1982), il nous a paru intéressant de définir les conditions optimales de température et d'humidité pour l'incubation et l'éclosion chez l'escargot Petit-gris, Helix aspersa. Ainsi, cette étude est effectuée à partir de pontes provenant, soit de populations de reproducteurs vivant dans diverses régions de France, soit d'escargots nés en élevage réalisé en bâtiment contrôlé. Enfin, divers types de «substrats» sont testés afin d'obtenir les meilleures conditions d'incubation des aufs et d'éclosion des jeunes.

\section{Techniques expérimentales et matériel utilisé}

\section{A. Protocole expérimental}

Pour cette étude, nous suivons un protocole expérimental, précédemment mis au point au laboratoire pour l'étude du bilan hydrique de divers Gastéropodes Pulmonés (Lazaridou-Dimitriadou \& Daguzan, 1978 ; Charrier \& Daguzan, 1980 ; HommaY, 1982). Nous effectuons ainsi vingt types d'expériences permettant de combiner 4 températures $\left(17,20,25,30^{\circ} \mathrm{C}\right)$ avec 5 taux d'humidité relative $(70,80,90$, 95 et 100 p. $100 \mathrm{Hr}$ ).

\section{Pots de ponte et substrat d'incubation}

Les pots utilisés pour recueillir les pontes des escargots sont des pots de terre cuite $(\varnothing: 7 \mathrm{~cm} ; \mathrm{h}: 6 \mathrm{~cm})$ utilisés par les pépiniéristes et renfermant du terreau horticole stérilisé (GuéméNÉ \& DaguZan, 1982).

Chaque pot est recouvert dune plaque de plexiglass afin de recueillir les nouveaunés qui s'y collent après leur éclosion (DaGUZAN, 1981).

\section{Les enceintes expérimentales ou «incubateurs »}

Les pots, contenant les pontes, sont placés dans des boîtes en plexiglas $(24 \mathrm{~cm}$ $\times 18 \mathrm{~cm} \times 10 \mathrm{~cm})$ transparentes et fermées hermétiquement par un couvercle dont les bords sont enduits d'une graisse au silicone, spéciale pour vide. Afin de renouveler l'air contenu dans les enceintes, tous les matins, les couvercles sont ôtés pendant quelques minutes.

Ces boîtes sont munies d'un faux plancher fixé à $2,5 \mathrm{~cm}$ de la base et constitué d'une armature plastique recouverte d'une toile de nylon perméable à mailles carrées servant de support pour les pots de ponte.

(1) Moyenne \pm erreur-standard. 


\section{Contrôle de la température}

Les différentes températures choisies sont obtenues en plaçant les enceintes expérimentales dans des armoires climatisées, thermostatées type «Jouan» munies de portes vitrées.

\section{Contrôle de l'humidité relative}

Les divers taux d'humidité relative sont obtenus grâce à l'action déshydratante de solutions aqueuses de soude. La méthode consiste à utiliser des concentrations variables de soude afin d'obtenir les densités correspondant aux pressions de vapeur d'eau désirées.

Les diverses solutions aqueuses de soude sont réalisées en se référant aux normes établies par Solomon (1951) et MADGE (1961). Une humidité relative de 100 p. 100 est réalisée en plaçant uniquement de l'eau distillée au fond des enceintes. Le contrôle des densités après plusieurs mois d'expérience montre que les diverses solutions sont stables.

Chaque boîte contient $600 \mathrm{ml}$ de solution, dont la surface d'échange est de $432 \mathrm{~cm}^{2}$. Ces boîtes sont alors placées, quelques heures avant les expériences à la température désirée afin d'obtenir l'équilibre thermohygrométrique souhaité. Il faut 4 heures pour que l'équilibre d'une solution à 90 p. $100 \mathrm{de} \mathrm{Hr}$ soit atteint à la température de $20^{\circ} \mathrm{C}$. Toutefois, la mesure de l'humidité relative à l'aide d'un thermohygromètre «Rotronic Hygroskop » montre, qu'après une heure, le taux est déjà de 80 p. $100 \mathrm{Hr}$.

\section{Contrôle de la photopériode}

Les diverses enceintes climatiques présentant des portes vitrées, les escargots sont soumis à la photopériode naturelle qu'on enregistre dans le local possédant de grandes baies vitrées.

\section{B. Matériel biologique et méthode d'étude}

Les pontes utilisées sont obtenues au cours de la reproduction d'escargots Petitsgris provenant, soit de la Bretagne (105 individus), soit de la Charente-Maritime (153 individus), soit enfin de l'élevage expérimental en bâtiment contrôlé du Centre Hélicole du Magneraud, près de Surgères, en Charente-Maritime (95 individus).

Près de 43000 œufs, dénombrés au cours de cette période de reproduction de 8 semaines, servent à la réalisation de cette étude. Il s'agit des œufs qui ont été collectés au cours du travail précédent relatif aux variations des accouplements et des pontes observées chez l'escargot Petit-gris (Helix aspersa) selon son origine géographique (GUÉMÉNÉ \& DAGUZAN, 1982).

Les oufs, après avoir été comptés et identifiés, sont disposés ou non dans un substrat (terreau), puis placés dans les incubateurs caractérisés chacun par un couple thermo-hygrométrique donné.

$\mathrm{Au}$ fur et à mesure que les nouveau-nés apparaissent, ils viennent se fixer sur les plaques de plexiglass. Ils sont alors comptabilisés et récupérés tous les matins. Ces 
diverses observations permettent ainsi de calculer le taux de natalité et les durées d'incubation et d'éclosion définis selon les normes établies par Daguzan (1981) :

\author{
nombre de jeunes éclos

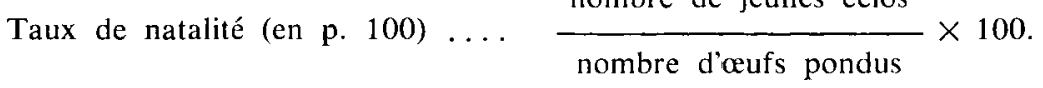 \\ Durée d'incubation (en jours) .... durée qui s'écoule entrc la «ponte» et \\ léclosion des œufs d'une ponte donnée. \\ Durée d'éclosion (en jours) ..... durée qui s'écoule entre le début et la fin \\ de l'éclosion d'une ponte donnée.
}

\title{
III. Résultats
}

Les différents résultats sont regroupés dans des tableaux (1 à 7) afin d'en faciliter la comparaison.

Dans un premier temps, nous essayons de montrer que l'origine des escargots a une influence sur l'incubation et l'éclosion (tabl. 1, 2, 3). Dans un second temps, nous tentons de voir si, la photopériode à laquelle sont soumis les reproducteurs d'une part, et la présence ou non de substrat incubateur d'autre part, ont une importance sur le développement des œufs (tabl. 4, 5). Enfin, il nous paraît intéressant de voir si l'incubation des cufs et l'éclosion des jeunes escargots varient selon la nature ct la forme des oufs (tabl. 6, 7).

Etant donné que linfluence du substrat incubateur, négative ou positive, et le temps nécessaire à son équilibration thermohygrométrique ne sont pas connus, nous admettons que, pour les expériences réalisées à l'aide d'un substrat donné, les taux d'humidité relative et les températures cho:sis correspondent effectivement aux valcurs thermohygrométriques ambiantes.

\section{Discussion}

\section{A. Influence de l'origine des escargots reproducteurs}

Quelle que soit l'origine géographique des géniteurs, les résultats sont comparables (tabl. 1 et 2). Les taux de natalité, bien qu'ils soient significativement différents entre eux, atteignent toujours des valeurs plus élevées aux conditions thermohygrométriques suivantes : $\left(20^{\circ} \mathrm{C}-95\right.$ p. $\left.100 \mathrm{Hr}\right),\left(20^{\circ} \mathrm{C}-100\right.$ p. $\left.100 \mathrm{Hr}\right)$ et $\left(25^{\circ} \mathrm{C}\right.$ 100 p. $100 \mathrm{Hr}$ ) pour les «Bretons », conditions auxquelles il faut y adjoindre celles de $\left(20^{\circ} \mathrm{C}-95\right.$ p. $\left.100 \mathrm{Hr}\right)$ et $\left(25^{\circ} \mathrm{C}-95\right.$ p. $\left.100 \mathrm{Hr}\right)$ pour les « Charentais».

Pour tous ces couples thermohygrométriques, le taux de natalité obtenu est toujours supérieur à 70 p. 100 . Il atteint respectivement 82 p. 100 (《Charentais 》) et $91,5$ p. 100 («Bretons $»)$ pour la thermohygrométrie $\left(20^{\circ} \mathrm{C}-100\right.$ p. $100 \mathrm{Hr}$ ) qui semble être la plus favorable. 
Tableau 1

Taux de natalité, durées d'incubation et d'éclosion pour des aufs d'Helix aspersa (Müller) originaires de Bretagne, en fonction de la thermohygrométrie (incubation dans terreau horticole).

Hatching rate, incubation and hatching lengths, obtained for eggs of Helix aspersa (Müller) from Brittany, according to the thermohygrometry

(incubation in horticultural vegetable mould).

\begin{tabular}{|c|c|c|c|c|c|c|}
\hline $\mathrm{T}{ }^{\prime \prime} \mathrm{C}$ & $\begin{array}{c}\mathrm{Hr} \% \\
\rightarrow\end{array}$ & 70 & 80 & 90 & 95 & 100 \\
\hline \multirow{4}{*}{$17^{\circ} \mathrm{C}$} & $\mathrm{N}$ & 461 & 376 & 528 & 421 & 320 \\
\hline & T.N. & 0,0 & 0,3 & 36,7 & 21,6 & 0,0 \\
\hline & D.I. & - & $(56)$ & $37,2 \pm 4,6$ & $50,5 \pm 12,6$ & - \\
\hline & D.E. & - & (1) & $10,0 \pm 5,5$ & $8,0 \pm 5,7$ & - \\
\hline \multirow{4}{*}{$20^{\circ} \mathrm{C}$} & $\mathbf{N}$ & 349 & 529 & 415 & 825 & 740 \\
\hline & T.N. & 14,0 & 2,5 & 26,0 & 71,8 & 91,5 \\
\hline & D.I. & $40,0 \pm 9,9$ & $34,3 \pm 11,3$ & $37,3 \pm 8,8$ & $19,9 \pm 0,9$ & $13,8 \pm 1,9$ \\
\hline & D.E. & $16,0 \pm 7,1$ & $8,0 \pm 6,0$ & $28,3 \pm 9,2$ & $13,3 \pm 4,9$ & $4,4 \pm 1,4$ \\
\hline \multirow{4}{*}{$25^{\circ} \mathrm{C}$} & $\mathrm{N}$ & 392 & 285 & 364 & 640 & 884 \\
\hline & T.N. & 0,3 & 0,0 & 13,0 & 15,2 & 91,0 \\
\hline & D.I. & (34) & - & $23,3 \pm 6,6$ & $14,7 \pm 3,2$ & $22,8 \pm 8,5$ \\
\hline & D.E. & (1) & - & $14,7 \pm 4,6$ & $8,8 \pm 1,6$ & $5,0 \pm 1,2$ \\
\hline \multirow{4}{*}{$30^{\circ} \mathrm{C}$} & $N$ & 543 & 564 & 692 & 267 & 371 \\
\hline & T.N. & 0,0 & 0,0 & 0,0 & 0,7 & 0,0 \\
\hline & D.I. & 一 & 一 & 一 & (31) & - \\
\hline & D.E. & 一 & - & - & (1) & - \\
\hline
\end{tabular}

$\mathrm{N}$ : Nombre d'œufs mis en incubation - Number of eggs to incubate.

T.N. : Taut de natalité (p. 100) - Hatching rate (p. 100).

D.I. : Durée incubation (j) - Incubation length.

D.E. : Durée éclosion (j) - Hatching length.

$(\overrightarrow{\mathrm{m}} \pm \mathrm{Sm})$. 


\section{TABLEAU 2}

Taux de natalité, durées d'incubation et d'éclosion pour des aufs d'Helix aspersa (Miiller) originaires de Charente-Maritime, en fonction de la thermohygrométrie

(incubation dans terrean horticole).

Hatching rate, incubation and hatching lengths, obtained for eggs of Helix aspersa (Miiller) form Charente-Maritime, according to the thermohygrometry (incubation in horticultural vegetable mould).

\begin{tabular}{|c|c|c|c|c|c|c|}
\hline $\mathrm{T}^{\circ} \mathrm{C}$ & $\begin{array}{c}\mathrm{Hr} \% \\
\rightarrow\end{array}$ & 70 & 80 & 90 & 95 & 100 \\
\hline $17^{\circ} \mathrm{C}$ & $\begin{array}{l}\text { N } \\
\text { T.N. } \\
\text { D.I. } \\
\text { D.E. }\end{array}$ & $\begin{array}{l}- \\
- \\
-\end{array}$ & - & $\begin{array}{l}- \\
- \\
-\end{array}$ & $\begin{array}{l}82 \\
10,1 \\
(40) \\
(20)\end{array}$ & $\begin{array}{c}115 \\
0,0 \\
- \\
- \\
\end{array}$ \\
\hline $20^{\circ} \mathrm{C}$ & $\begin{array}{l}\text { N } \\
\text { T.N. } \\
\text { D.I. } \\
\text { D.E. }\end{array}$ & $\begin{array}{c}546 \\
28,9 \\
18,0 \pm 5,9 \\
4.7 \pm 1.3\end{array}$ & $\begin{array}{c}629 \\
57,4 \\
16,7 \pm 1,2 \\
12,2 \pm 6,9\end{array}$ & $\begin{array}{c}1004 \\
78,9 \\
17,3 \pm 1,1 \\
5,3 \pm 2,3\end{array}$ & $\begin{array}{c}116.3 \\
74,7 \\
14,6 \pm 0,5 \\
2,5 \pm 0,6\end{array}$ & $\begin{array}{c}3535 \\
82,2 \\
12,3 \pm 0,5 \\
3,2 \pm 0.9\end{array}$ \\
\hline $25^{\circ} \mathrm{C}$ & $\begin{array}{c}\text { N } \\
\text { T.N. } \\
\text { D.I. } \\
\text { D.E. }\end{array}$ & $\begin{array}{c}378 \\
0,8 \\
(14) \\
(1)\end{array}$ & $\begin{array}{c}671 \\
42,3 \\
15,7 \pm 1,3 \\
5.3 \pm 2,0\end{array}$ & $\begin{array}{c}1089 \\
52,9 \\
14,5 \pm 0,8 \\
6,2 \pm 0,6\end{array}$ & $\begin{array}{c}983 \\
72,3 \\
12,7 \pm 0,2 \\
4.7 \pm 1.0\end{array}$ & $\begin{array}{c}478 \\
73,2 \\
12,2 \pm 0,4 \\
1,6 \pm 0,3\end{array}$ \\
\hline
\end{tabular}

TABLEAU 3

Taux de natalité, durées d'incubation et d'éclosion pour des aufs d'Helix aspersa (Müllor) élevés en bâtiment contrôlé au Centre helicole du Magneraud, près de Surgères

(Charente-Maritime), en fonction de la thermolygrométrie (incubation dans terreau horticole).

Hatching rate, incubation and hatching lengths, obtained for eggs of Helix aspersa (Müller) reared in heated building at the heliuculture center of Magneraud, near Surgères

(Charente-Maritime),

according to the thermohygrometry (incubation in horticultural vegetable mould).

\begin{tabular}{|c|c|c|c|c|c|c|}
\hline $\mathrm{T}^{\circ} \mathrm{C}$ & $\begin{array}{c}\mathrm{Hr} \% \\
\rightarrow\end{array}$ & 70 & 80 & 90 & 95 & 100 \\
\hline $17^{\circ} \mathrm{C}$ & $\begin{array}{l}\text { N } \\
\text { T.N. } \\
\text { D.I. } \\
\text { D.E. }\end{array}$ & - & $\begin{array}{l}- \\
-\end{array}$ & $\begin{array}{l}\text { - } \\
-\end{array}$ & $\begin{array}{c}120 \\
0,0 \\
- \\
-\end{array}$ & $\begin{array}{c}143 \\
0,0 \\
- \\
-\end{array}$ \\
\hline $20^{\circ} \mathrm{C}$ & $\begin{array}{l}\text { N } \\
\text { T.N. } \\
\text { D.I. } \\
\text { D.E. }\end{array}$ & $\begin{array}{c}480 \\
15,0 \\
21,5 \pm 2,5 \\
6,0 \pm 4,0\end{array}$ & $\begin{array}{c}425 \\
19,1 \\
17,3 \pm 1,8 \\
3,3 \pm 1,8\end{array}$ & $\begin{array}{c}412 \\
85,8 \\
17,7 \pm 2,3 \\
4,2 \pm 1,3\end{array}$ & $\begin{array}{c}577 \\
78,0 \\
14,3 \pm 0,4 \\
3,0 \pm 0,7\end{array}$ & $\begin{array}{c}408 \\
96,6 \\
14,7 \pm 2,0 \\
2,2 \pm 0,8\end{array}$ \\
\hline $25^{\circ} \mathrm{C}$ & $\begin{array}{c}\text { N } \\
\text { T.N. } \\
\text { D.I. } \\
\text { D.E. }\end{array}$ & $\begin{array}{c}152 \\
0,0 \\
- \\
-\end{array}$ & $\begin{array}{c}201 \\
0,0 \\
- \\
-\end{array}$ & $\begin{array}{c}258 \\
5,4 \\
16,5 \pm 2,1 \\
6,0 \pm 1,4\end{array}$ & $\begin{array}{c}714 \\
33,0 \\
15,0 \pm 1,0 \\
5,2 \pm 1,6\end{array}$ & $\begin{array}{c}244 \\
71,7 \\
13,3 \pm 1,1 \\
4,7 \pm 2,5\end{array}$ \\
\hline
\end{tabular}

(Voir légende page ci-contre.) 


\section{TABLEAU 4}

Taux de natalité, durées d'incubation et d'éclosion pour des aufs d'Helix aspersa (Müller) originaires de Charente-Maritime et élevés en pénombre continue (1 lux), en fonction de la thermohygrométrie (incubation dans terreau horticole).

Hatching rate, incubation and hatching lengths, obtained for eggs of Helix aspersa (Müller) from Charente-Maritime and reared in continual penumbra (1 lux) according to the thermohygrometry (incubation in horticultural vegetable mould).

\begin{tabular}{c|c|c|c|c|c|c}
\hline \hline $\mathrm{T}{ }^{\circ} \mathrm{C}$ & $\begin{array}{c}\mathrm{Hr} \% \\
\rightarrow\end{array}$ & 70 & 80 & 90 & 95 & 100 \\
\cline { 2 - 6 } $20^{\circ} \mathrm{C}$ & $\mathrm{N}$ & 236 & 173 & 306 & 705 & 219 \\
& T.N. & 3,4 & 74,0 & 51,0 & 45,2 & 85,9 \\
& D.I. & $(30)$ & $15,0 \pm 4,0$ & $19,5 \pm 3,5$ & $15,6 \pm 1,3$ & $12,0 \pm 1,4$ \\
& D.E. & $(2)$ & $4,1 \pm 1,1$ & $10,0 \pm 8,5$ & $2,4 \pm 0,9$ & $1,5 \pm 0,7$ \\
\hline \multirow{5}{*}{$25^{\circ} \mathrm{C}$} & $\mathrm{N}$ & - & - & 104 & 100 & 261 \\
& T.N. & - & - & 76,0 & 69,0 & 80,1 \\
& D.I. & - & - & $13,0 \pm 0,5$ & $12,0 \pm 0,3$ & $12,0 \pm 0,7$ \\
& D.E. & - & - & $6,2 \pm 0,4$ & $4,0 \pm 0,5$ & $1,7 \pm 0,4$ \\
\hline
\end{tabular}

Tableau 5

Taux de natalité, durées d'incubation et d'éclosion pour des aufs d'Helix aspersa (Müller) originaires de Charente-Maritime, en fonction de la thermohygrométrie (incubation en absence de substrat).

Hatching rate, incubation and hatching lengths, obtained for eggs of Helix aspersa (Müller) from Charente-Maritime, according to the thermohygrometry (incubation in absence from substratum).

\begin{tabular}{|c|c|c|c|c|c|c|}
\hline $\mathrm{T}^{\circ} \mathrm{C}$ & $\begin{array}{c}\mathrm{Hr} \% \\
\rightarrow\end{array}$ & 70 & 80 & 90 & 95 & 100 \\
\hline $17^{\circ} \mathrm{C}$ & $\begin{array}{l}\text { N } \\
\text { T.N. } \\
\text { D.I. } \\
\text { D.E. }\end{array}$ & $\begin{array}{l}- \\
- \\
-\end{array}$ & $\begin{array}{c}- \\
-\end{array}$ & $\begin{array}{c}- \\
-\end{array}$ & $\begin{array}{c}286 \\
0,0 \\
- \\
-\end{array}$ & $\begin{array}{c}338 \\
72,8 \\
15,8 \pm 1,2 \\
2,6 \pm 1,3 \\
\end{array}$ \\
\hline $20^{\circ} \mathrm{C}$ & $\begin{array}{l}\text { N } \\
\text { T.N. } \\
\text { D.I. } \\
\text { D.E. }\end{array}$ & $\begin{array}{c}395 \\
0,0 \\
- \\
-\end{array}$ & $\begin{array}{c}562 \\
0,0 \\
- \\
-\end{array}$ & $\begin{array}{c}401 \\
0,0 \\
- \\
-\end{array}$ & $\begin{array}{c}500 \\
30,0 \\
9,7 \pm 0,7 \\
3,0 \pm 0,9\end{array}$ & $\begin{array}{c}901 \\
63,3 \\
9,4 \pm 0,4 \\
2,7 \pm 0,4\end{array}$ \\
\hline $25^{\circ} \mathrm{C}$ & $\begin{array}{l}\text { N } \\
\text { T.N. } \\
\text { D.I. } \\
\text { D.E. }\end{array}$ & $\begin{array}{l}- \\
- \\
-\end{array}$ & $\begin{array}{l}- \\
-\end{array}$ & - & $\begin{array}{c}428 \\
14,0 \\
11,6 \pm 1,2 \\
1,2 \pm 0,2\end{array}$ & $\begin{array}{c}471 \\
13,2 \\
12,8 \pm 1,4 \\
1,2 \pm 0,2\end{array}$ \\
\hline
\end{tabular}

$\mathrm{N}$ : Nombre d'œufs mis en incubation - Number of eggs to incubate.

T.N. : Taut de natalité (p. 100) - Hatching rate (p. 100).

D.I. : Durée d'incubation (j) - Iucubation length.

D.E. : Durée d'éclosion (j) - Hatching length. $(\overline{\mathrm{m}} \pm \mathrm{Sm})$ 


\section{TABLEAU 6}

Comparaison entre l'incubation d'aufs translucides et d'æufs opaques d'Helix aspersa (Müller) selon l'origine des géniteurs et le substrat d'incubation.

Comparison between the incubation of translucent eggs and that of opaque eggs of Helix aspersa (Müller) according to the origin of the breeding snails and the incubation substrate.

\begin{tabular}{|c|c|c|c|}
\hline $\begin{array}{l}\text { Origine - Substrat } \\
\text { Thermohygrométrie } \\
\text { Thermohygrometry }\end{array}$ & $\begin{array}{l}\text { Type d'œufs } \\
\text { Paramètres } \\
\text { Parameters }\end{array}$ & $\begin{array}{l}\text { Translucides } \\
\text { Translucent }\end{array}$ & $\begin{array}{c}\text { Opaques } \\
\text { Opaque }\end{array}$ \\
\hline \multirow{5}{*}{$\begin{array}{c}\text { "Charentais } \\
\text { sans substrat } \\
\text { without substrate } \\
25^{\circ} \mathrm{C}-95 \% \mathrm{Hr}\end{array}$} & $\begin{array}{l}\text { Nombre d'œufs (N) } \ldots \ldots \ldots \\
\text { Number of eggs }\end{array}$ & 152 & 276 \\
\hline & $\begin{array}{l}\text { Taux de natalité }(\%) \ldots \ldots \ldots \\
\text { Hatching rate }\end{array}$ & 6,6 & 18,1 \\
\hline & Durée d'incubation (j) .. & $12,0 \pm 2,8$ & $11,3 \pm 1,7$ \\
\hline & Incubation length (d) & & \\
\hline & $\begin{array}{l}\text { Durée d'éclosion (j) } \ldots \ldots \ldots \\
\text { Hatching length (d) }\end{array}$ & $1,0 \pm 0,0$ & $1,3 \pm 0,4$ \\
\hline \multirow{4}{*}{$\begin{array}{c}\text { «Charentais } \\
\text { sans substrat } \\
\text { without substrate } \\
20^{\circ} \mathrm{C}-100 \% \mathrm{Hr}\end{array}$} & $\begin{array}{l}\text { Nombre d'œufs (N) } \ldots \ldots \ldots \\
\text { Number of eggs }\end{array}$ & 86 & 845 \\
\hline & $\begin{array}{l}\text { Taux de natalité }(\%) \ldots \ldots \ldots \\
\text { Hatching rate }\end{array}$ & 62,8 & 64,4 \\
\hline & $\begin{array}{l}\text { Durée d'incubation (j) } \ldots \ldots \ldots \\
\text { Incubation length }(d)\end{array}$ & $10,5 \pm 2,1$ & \\
\hline & $\begin{array}{l}\text { Durée d'éclosion }(\mathrm{j}) \ldots \ldots \ldots \\
\text { Hatching length }(d)\end{array}$ & $1,0 \pm 0,0$ & $3,1 \pm 0,4$ \\
\hline \multirow{4}{*}{$\begin{array}{c}\text { «Magneraud } \\
\text { substrat : terreau horticole } \\
\text { substrate : vegetable mould } \\
25{ }^{\circ} \mathrm{C}-100 \% \mathrm{Hr}\end{array}$} & $\begin{array}{l}\text { Nombre d'œufs (N) } \ldots \ldots \ldots \\
\text { Number of eggs }\end{array}$ & 99 & 145 \\
\hline & $\begin{array}{l}\text { Taux de natalité }(\%) \ldots \ldots \ldots \\
\text { Hatching rate }\end{array}$ & 42,4 & 92,0 \\
\hline & $\begin{array}{l}\text { Durée d'incubation (j) } \cdots \cdots \\
\text { Incubation length }(d)\end{array}$ & $13,5 \pm 2,1$ & $(13,0)$ \\
\hline & $\begin{array}{l}\text { Durée d'éclosion }(\mathrm{j}) \ldots \ldots \ldots \\
\text { Hatching length }(d)\end{array}$ & $4,5 \pm 4,9$ & $(5,0)$ \\
\hline
\end{tabular}




\section{TABLEAU 7}

Comparaison entre l'incubation d'aufs sphériques et celle d'aufs ovoüdes $d$ 'Helix aspersa (Mïller)

originaires de Bretagne (incubation dans terreau horticole à $20^{\circ} \mathrm{C}$ et $95 \% \mathrm{Hr}$ ).

Comparison between incubation of spherical eggs and ovoid eggs

of Helix aspersa (Miiller) coming from Brittany

(incubation in horticultural vegetable mould; $20^{\circ} \mathrm{C} ; 95 \% \mathrm{Hr}$ ).

\begin{tabular}{|c|c|c|}
\hline $\begin{array}{l}\text { Type d'œufs } \\
\text { Type of eegs } \\
\text { Paramètres }\end{array}$ & $\begin{array}{l}\text { Eufs sphériques } \\
\text { Spherical eggs }\end{array}$ & $\begin{array}{l}\text { Eufs ovoïdes } \\
\text { Ovoid eggs }\end{array}$ \\
\hline $\begin{array}{l}\text { Nombre d'œufs }(\mathrm{N}) \ldots \ldots \ldots \ldots \ldots \ldots \ldots \\
\text { Number of eggss }\end{array}$ & 129 & 80 \\
\hline $\begin{array}{l}\text { Taux de natalité }(\%) \ldots \ldots \ldots \ldots \ldots \ldots \\
\text { Hatching rate }\end{array}$ & 61,2 & 26,2 \\
\hline $\begin{array}{l}\text { Durée d'incubation (jours) } \ldots \ldots \ldots \ldots \ldots \\
\text { Incubation length (days) }\end{array}$ & $17,5 \pm 3,5$ & $17,5 \pm 3,5$ \\
\hline $\begin{array}{l}\text { Durée d'éclosion (jours) } \ldots \ldots \ldots \ldots \ldots \ldots \\
\text { Hatching length (days) }\end{array}$ & $9,0 \pm 4,2$ & $10,0 \pm 5,6$ \\
\hline
\end{tabular}

Des essais à $30^{\circ} \mathrm{C}$ sont effectués pour les «Bretons»; les résultats s'avérant négatifs, nous n'avons pas jugé bon de les reproduire pour les autres populations. Après observations dans la terre, nous constatons que des oufs ont éclos, mais que les jeunes formés sont morts avant de monter à la surface.

Pour les divers taux d'hygrométrie utilisés à la température de 17 " $\mathrm{C}$, le taux de natalité est nul. Il faut remarquer cependant que pour les «Charentais », nous n'avons expérimenté que sur une seule ponte pour chaque hygrométrie, ce qui n'est pas suffisant pour affirmer un résultat.

Les durées dincubation pour les conditions optimales présumées déjà citées sont comprises entre 12 et 23 jours. Les autres conditions n'offrent pas de meilleures perspectives. Si cette durée passe du simple au double, du fait de sa grande variabilité suivant les pontes, la différence n'est pas significative. Il s'avère que les meilleures conditions sont : $(20$ "C -100 p. $100 \mathrm{Hr}),(25$ "C -95 p. $100 \mathrm{Hr})$ pour les «Bretons », et $\left(20{ }^{\circ} \mathrm{C}-95\right.$ p. $\left.100 \mathrm{Hr}\right),\left(20{ }^{\prime C} \mathrm{C}-100\right.$ p. $\left.100 \mathrm{Hr}\right),(25$ "C $-90,95$ et 100 p. $100 \mathrm{Hr})$, pour les «Charentais».

En ce qui concerne les durées d’éclosion, les plus brèves sont enregistrées pour les conditions thermohygrométriques $\left(20^{\circ} \mathrm{C}-100 \mathrm{p}\right.$. $\left.100 \mathrm{Hr}\right): 4$ jours et $\left(25^{\circ} \mathrm{C}\right.$ 100 p. $100 \mathrm{Hr}): 5$ jours chez les «Bretons $;\left(20^{\circ} \mathrm{C}-95\right.$ et 100 p. $\left.100 \mathrm{Hr}\right): 2$ à 3 jours et $(25$ "C -100 p. $100 \mathrm{Hr}): 2$ jours chez les «Charentais», les différences avec d'autres conditions n'étant d'ailleurs pas toujours significatives. 
Ainsi, on note que le taux de natalité enregistré pour les œufs d'escargots «Bretons » est supérieur d'environ 10 p. 100 à celui qu'on obtient pour les œufs provenant de «Charentais », à la thermohygrométrie de $\left(20^{\circ} \mathrm{C}-100\right.$ p. $\left.100 \mathrm{Hr}\right)$ qui correspond aux conditions optimales des deux populations. Par contre, il semble que les pontes issues de «Charentais » soient plus résistantes aux conditions défavorables; en effet, la gamme des taux d'humidité relative ambiante où les éclosions sont nombreuses, est plus étendue.

Ainsi, quelle que soit l'origine géographique des escargots Petits-gris reproducteurs, les conditions thermohygrométriques optimales pour l'incubation des œufs et l'éclosion des jeunes sont : une température de $20^{\circ} \mathrm{C}$ associée à une humidité relative de 100 p. $100 \mathrm{Hr}$.

\section{B. Influence de la captivité des escargots reproducteurs}

Si on compare les pontes provenant d'escargots «charentais » sauvages (tabl. 3) avec celles obtenues à partir de Petits-gris de première génération, nés en bâtiment d'élevage, au Centre Hélicicole du Magneraud (tabl. 3), on constate que les gammes thermohygrométriques favorables à l'incubation des œufs sont identiques.

Cependant, si le taux de natalité est maximum pour les mêmes conditions $\left(20^{\circ} \mathrm{C}\right.$ 100 p. $100 \mathrm{Hr}$ ), il s'élève cependant à 96,6 p. 100 pour les « Magneraud », soit 14 p. 100 de plus que pour les «Charentais» $(82$ p. 100).

Ainsi, il est possible d'affirmer que la captivité des escargots reproducteurs en élevage ne semble pas entraîner une diminution du taux de natalité, ce qui confirme les résultats obtenus par DaGUZAN (1981).

\section{Influence de la lumière sur les reproducteurs}

Au cours de l'étude précédente (Guemene \& Daguzan, 1982), nous avons observé que les escargots qui vivaient en pénombre constante pondaient autant que ceux qui étaient soumis à un éclairage permanent. Il nous a donc paru intéressant de voir si le fait d'élever des reproducteurs en obscurité quasi-totale (1 lux) n'avait pas un effet inhibiteur sur la fécondité des oufs et n'entraînerait pas une diminution du taux de mortalité (tabl. 4) par rapport aux résultats obtenus pour des escargots reproducteurs soumis à une photo-période naturelle (tabl. 2).

Les résultats sont très disparates et ne permettent pas de conclure à une quelconque influence de ce facteur lumière.

\section{Influence de l'absence de «substrat incubateur»}

Cette expárience est réalisée avec des oufs pondus par des Petits-gris «Charentais $\gg($ tabl. 6). Pour cette étude, les pontes sont disposées sous récipient de verre $(\varnothing: 6 \mathrm{~cm} ; \mathrm{h}: 7 \mathrm{~cm})$ retourné et placé sur de la mousse plastique humidifiée, jouant le rôle de cloche.

Les essais effectués à $20^{\circ} \mathrm{C}$ et à 70,80 et $90 \mathrm{p}$. $100 \mathrm{Hr}$, montrent que les pontes se dessèchent toutes dans leur ensemble respectivement les $4^{e}, 5^{\circ}$ et $6^{\circ}$ jour d'incubation. 
Pour une humidité relative de 95 p. $100 \mathrm{Hr}$, le taux de natalité est nul à $17^{\circ} \mathrm{C}$, de 30 p. 100 à $20^{\circ} \mathrm{C}$ et de 14 p. 100 à $25^{\circ} \mathrm{C}$, les aufs restant se desséchant.

Pour un taux d'humidité saturée, le taux de natalité est de 72,8 p. 100 à $17^{\circ} \mathrm{C}$ et de 63,3 p. 100 à $20^{\circ} \mathrm{C}$. Les durées d'incubation sont respectivement de 16 jours à $17^{\circ} \mathrm{C}$ et de 9 jours à $20^{\circ} \mathrm{C}$ pour une durée moyenne d'éclosion de 3 jours environ.

Enfin, il s'avère qu'un élément vient contrarier cette étude, il s'agit d'une condensation sous la cloche qui entraîne le «pourrissement» des pontes à $20^{\circ} \mathrm{C}$ et surtout à $25^{\circ} \mathrm{C}$.

$\mathrm{Si}$ nous considérons les taux de natalité maximum, on constate qu'ils sont plus faibles en absence de substrat, ceci étant sans doute dû au mode d'utilisation des pots en verre.

Pour des taux d'humidité relative inférieurs ou égaux à 90 p. 100 , il n'y a pas d'éclosion si les oufs ne sont pas protégés par un substrat. On serait donc tenté d'affirmer que les durées d'incubation sont plus brèves en absence de substrat, mais en fait les jeunes escargots ne remontent pas immédiatement après l'éclosion à la surface de la terre. Les nouveau-nés séjournent dans leur nid durant 3 à 6 jours (HerzbERG \& Herzberg, 1962). Cette période d'attente est-elle importante pour la croissance et la maturité sexuelle ultérieures ?

\section{E. Eclosion des oufs selon leur nature et leur forme}

\section{Les aufs roses}

Nous avons pu démontrer que les œufs de l'escargot Petit-gris, Helix aspersa, varient selon leur forme et leur couleur (Guemene \& Daguzan, 1982). Ainsi, ils sont le plus souvent sphériques, mais quelquefois ovoïdes ou doubles. Leur couleur est en général blanc opaque, mais certains sont translucides. Enfin, des œufs peuvent être roses : dans ce cas, ils sont parasités par un champignon (Fusarium sp.) (MeYNADier, AMARgier \& Vey, 1977).

Les œufs roses n'aboutissent jamais à la formation de jeunes, observation qui est conforme à celle de Meynadier et al. (1977). Il est d'ailleurs intéressant de savoir que la contamination expérimentale est irréalisable ; aussi le fait d'avo:r quelques oufs roses n'est-il pas préjudiciable pour le reste de la ponte.

\section{Les oufs translucides et les aufs opaques}

Les expériences se limitent à l'observation de quelques pontes provenant de Petits-gris "Charentais» sauvages ou d'escargots de première génération obtenus en élevage au Magneraud (tabl. 6).

Si les durées d'incubation et d'éclosion sont similaires pour les deux types d'œufs, le taux de natalité est significativement inférieur pour les oufs ovoïdes dans les lots expérimentés. Charrier (1980) a calculé des pourcentages différents d'œufs ovoïđes dans la nature et au laboratoire. Il sera intéressant de connaître la raison d'être de ces œufs si notre observation se vérifiait sur un plus grand nombre d'échantillons.

Il n'est possible de faire figurer des résultats que pour trois couples thermohygrométriques : $20^{\circ} \mathrm{C}-100$ p. $\left.100 \mathrm{Hr}\right),\left(25^{\circ} \mathrm{C}-95\right.$ p. $\left.100 \mathrm{Hr}\right)$ et $\left(25^{\circ} \mathrm{C}-100\right.$ p. $\left.100 \mathrm{Hr}\right)$. 
Le nombre de pontes translucides n'est que de deux pour chaque couple, ce qui est insuffisant pour en tirer des conclusions irréfutables.

A $\left(25^{\circ}\right.$ C -95 p. 100 ou 100 p. $\left.100 \mathrm{Hr}\right)$, ce taux est à chaque fois significativement inférieur pour les œufs translucides.

Les durées d'incubation et d'éclosion sont assez voisines. Il semble donc que la couche coquillée joue le rôle d'un bouclier dont l'absence dans des conditions plus défavorables s'avère préjudiciable, cette conclusion devant toutefois être confirmée.

\section{Les aufs doubles}

Sans avoir de résultats chiffrés, il est sûr que pour certains d'entre eux, les deux œufs «siamois» ont éclos. En effet, nous avons calculé un taux de natalité supérieur à 78 p. 100 pour une ponte composée à 60 p. 100 d'œufs doubles.

L'intérêt de cette observation réside dans le fait que nous pouvons imaginer ultérieurement l'utilisation de ces «jumeaux» pour des expériences comparatives.

\section{Les aufs sphériques et les aufs ovoïdes}

Les œufs d'une même ponte n'étant jamais tous ovoïdes, il est possible d'isoler les deux types d'oufs en fonction de leur forme (sphérique ou ovoïde) pour deux pontes de «Bretons» et de les incuber parallèlement dans des conditions similaires (tabl, 7).

On constate surtout que le taux de natalité est nettement plus élevé pour les œufs sphériques $(61,2$ p. 100) que pour ceux qui sont ovoïdes $(26,2$ p. 100). En ce qui concerne les durées d'incubation et d'éclosion, elles sont identiques quelle que soit la forme de l'œuf.

\section{Conclusion}

Le taux de natalité est sans doute un des paramètres les plus importants en Héliciculture. Toutefois, les durées d'incubation et d'éclosion sont également des critères qui doivent retenir l'attention. Il ne faut pas en effet que les jeunes mettent trop de temps à éclore, car l'immobilisation d'un instrument de travail est toujours préjudiciable. De même, l'éclosion d'une ponte en ordre dispersé a pour conséquence d'augmenter le temps de travail. De plus, si les jeunes mangent la coquille de leur ouf à la naissance (GASPARD, 1924), ils peuvent également manger les œufs non éclos se trouvant autour d'eux, la durée d'éclosion intervenant alors sur le taux de natalité.

Le couple thermohygrométrique $\left(20^{\circ} \mathrm{C}-100 \mathrm{p}\right.$. $\left.100 \mathrm{Hr}\right)$ est le plus satisfaisant pour l'incubation dans le terreau horticole quel que soit le critère de jugement (taux, durées ou origine des reproducteurs).

Cette stabilité expérimentale ne sera pas toujours reproductible à grande échelle (élevage). A cette fin, nous pouvons définir une gamme optimale plus étendue à l'intérieur de laquelle les taux de natalité et les durées d'incubation et d'éclosion sont également satisfaisants. La température devra être comprise entre 20 et $25^{\circ} \mathrm{C}$ et le taux d'humidité relative ambiante entre 95 et 100 p. $100 \mathrm{Hr}$. 
Toutefois, à une température de haut de gamme, nous ne pourrons associer qu'un fort taux d'humidité alors qu'une baisse de la température permet d'être moins exigeant quant aux taux d'humidité relative.

Des éclosions sont constatées en absence de substrat; sa présence n'est donc pas obligatoire. Les durées d'incubation et d'éclosion sont alors plus brèves, et si le taux de natalité est plus faible, notre façon de manipuler en est sans doute la cause... Le substrat, en jouant le rôle protecteur, permet par contre d'élargir la gamme thermohygrométrique utilisable.

Enfin, il serait peut-être intéressant de voir l'effet de la lumière et de la longueur d'onde sur le développement embryonnaire des escargots, de telles expériences ayant été menées par certains auteurs chez d'autres Pulmonés.

Accepté pour publication en mai 1983.

\section{Summary}

Variations in the reproductive capacities of «Petit-gris» snails (Helix aspersa Müller) according to their geographic origin

II - Incubation of eggs and hatching of juveniles

This study showed that the incubation length of eggs and hatching length of juveniles from «Petit-gris» snails (Helix aspersa Müller) kept in heated houses with controlled hygrometry was independent of the origin of the breeding snails and of the type of lighting used during the reproductive period.

Whatever the geographic origin (Brittany or Charentes) of the breeding snails, the optimum hygrometric conditions for incubation and hatching were a temperature of $20^{\circ} \mathrm{C}$ and a relative moisture of $100 \mathrm{p}$. 100 . Moreover, the hatching rate recorded in the above mentioned conditions for the eggs from «Bretons» snails was 10 p. 100 higher than that obtained with the eggs from «Charentes» snails.

It may be assert that raising breeding snails in captivity does not reduce the hatching rate, at least for the animals of the 1 st generation, but even seems to improve it.

The presence of substrate (vegetable mould) for incubation is not compulsory : without substrate the incubation and hatching lengths were much shorter. However, new-born snails did not climb immediately after hatching to the surface of the earth and it may be asked whether this waiting period is important for the further growth and sexual maturity of the juveniles.

\section{Références bibliographiques}

BagnaRd M., BondoIs J.L., 1976. L'escargot, son réseau industriel et commercial, sa biologie et son écologie, son élevage. Mémoire de fin d'étude I.S.A.R.A., édit. I.T.A.V.I., Paris, 150 p.

BASINGER A.J., 1931. The european brown snail in California. Rep. Calif. Agric. exper. Stat. Bull., 515, University of California, Printing office, Berkeley Californy, $20 \mathrm{p}$.

CARriK R., 1942. The grey field slug Agriolimax agrestis L. and its environnement. Ann. appl. Biol., 29, 43-55. 
Charrier M., 1980. Contribution à la biologie et à l'écophysiologie de l'escargot Petit-gris : Helix aspersa Müller (Gastéropode Pulmoné Stylommatophore). Thèse Doctorat de $3^{\text {* }}$ cycle, Rennes, $330 \mathrm{p}$.

Charrier M., Daguzan J., 1980. Etude du bilan hydrique et de son évolution en fonction de la température et de l'humidité relative chez Helix aspersa Müller (Mollusque Gastéropode Pulmoné). Haliotis, 10, 33-36.

Daguzan J., 1981. Contribution à l'élevage de l'escargot Petit-gris : Helix aspersa Müller (Mollusque Gastéropode Pulmoné stylommatophore). I - Reproduction et éclosion des jeunes, en bâtiment et en conditions thermohygrométriques contrôlées. Ann. Zootech., 30, 249-272.

Gaspard B., 1924. Beitrage zur Physiologie der Gartenschnecke (Helix pomatia L.). Dtsch. Arch. Physiol., 8, 243-290.

Guemene D., Daguzan J., 1982. Variations des capacités reproductrices de l'escargot Petitgris, Helix aspersa Müller (Mollusque Gastéropode Pulmoné Stylommatophore) selon son origine géographique. I - Accouplement et ponte. Ann. Zootech., 31, 369-390.

Herzberg F., Herzberg A., 1962. Observations on reproduction in Helix aspersa. Am. Midl. Nat., 68, 297-306.

Hоммау G., 1982. Importance de la thermohygrométrie chez l'escargot Petit-gris. Helix aspersa Müller. Recherches écophysiologiques sur l'action de Molluscicides à base de métaldéhyde chez les escargots et les limaces. Rapport d'activité, contrat Lonza, C.R.E.B.S., Université de Rennes, $\mathrm{n}^{*} 1,17$ p.

Lazaridou-Dimitriadou M., Daguzan J., 1978. Etude du bilan hydrique et de son évolution en fonction de la température et de l'humidité relative chez Euparypha pisana (Müller). Arch. Zool. exp. gen., 119, 549-564.

MADGE D.S., 1961. The control of relative humidity with aqueous solutions of sodium hydroxyde. Entomol. Exp. Appl., 4, 113-120.

Meynadier G., Amargier A., Vey A., 1977. Etude de la maladie des «pontes roses» du gastéropode Helix aspersa. Haliotis, 8, 265-270.

Pollard E., 1975. Aspects of the ecology of Helix pomatia L. J. anim. Ecol., 44, 305-329.

Solomon M.E., 1951. Control of humidity with $\mathrm{koH}, \mathrm{H}_{2} \mathrm{SO}_{4}$ and other solutions. Bull. entomol. Res., 42, 543-559.

Vincent C., Deray A., Enee J., Clerc M., Jugand M., Jugand F., Bolzoni D., 1982. Nombre d'œufs par ponte, durée d'incubation et croissance en laboratoire de Helix pomatia L. (Gastéropoda Pulmonata). Haliotis, 12, 13-20. 